\title{
4 Linked open data and aggregation infrastructure in the cultural heritage sector
}

\author{
A case study of $\mathrm{SOCH}$, a linked \\ data aggregator for Swedish \\ open cultural heritage
}

\author{
Marcus Smith
}

\section{Introduction}

Publishing openly licensed data is increasingly becoming the norm for research project outputs, museum and archive collections, monuments registers and more within the cultural heritage sector. Going a step further, this approach is often combined with making the data machine-readable on the semantic web as Linked Data, such that it can be queried and reused on a technical level as well as related to other data sets. This represents a change in mindset for how heritage data is published and disseminated, as well as for public engagement, institutional collaboration and how research is carried out.

Both of these changes - towards open licenses and Linked Data across systems - can be seen as a response to preexisting problems with the field of cultural heritage data. With digitisation, new questions of the copyright of scanned material have arisen - some as yet unresolved - which can lead to representations of a shared heritage being made inaccessible and unusable by most people. At the same time, the increased use of digital tools, workflows, and datastores have resulted in a plethora of closed, siloed systems that frequently record information about the same sorts of things, but can't talk to one another, and use incompatible terms and technologies. Thus, linked open data (LOD), with its emphasis on explicit open licensing, links between data sets, and interoperability through shared standards and vocabularies, is in many ways a natural fit.

This chapter aims to present an (incomplete) overview of the theory and practice of LOD in cultural heritage from a Swedish perspective, introducing key concepts and standards. We will begin by presenting an introduction to LOD and metadata aggregation as philosophical and technical concepts within digital cultural heritage, before providing an in-depth case study of the Linked Data platform Swedish Open Cultural Heritage (SOCH) at the 
Swedish National Heritage Board, which aggregates and publishes open metadata from Swedish heritage institutions and provides a technical interface for software developers to build applications. Then follow some reflections over the state of linked cultural heritage through the lens of the case study as presented, as well as challenges for the future. Since many new concepts, standards and acronyms will be introduced, a short glossary of terms is also included, with links for further reading.

\section{Background: Linked Data aggregation}

\section{LOD and FAIR principles}

The idea of LOD is described by a set of principles, both philosophical and technical, first articulated by Sir Tim Berners-Lee as a logical extension of the World Wide Web (see e.g., Berners-Lee 2006). The web can be seen as a distributed, decentralised directed graph: a network of documents - web pages - as nodes/vertices connected by hyperlinks as edges/arcs pointing from source to target, across a multitude of systems and domains with no single point of control. With Linked Data, rather than a web of linked documents, there is a web of semantically linked machine-readable data. "Semantically linked", in the sense that it is significant not only whether there is a link between two nodes in the web and in what direction it points, but also what the nature of that link - or relation - is. We might wish to assert, e.g., that the node representing an artefact was found at the node in the web representing a particular site, is depicted by the node for a photograph, described by a report etc. In this way, the web of data is at a very basic level composed of simple three-part assertions of the form subject $\rightarrow$ predicate $\rightarrow$ object, connecting two nodes - the subject and object - using a relation - the predicate. Just as HTML is the standard of interchange used to describe the web of documents we're familiar with, the Resource Description Framework (RDF; see Schreiber and Raimond 2014 ) is the model used to describe the web of data, and just like HTML, it uses IR Is as identifiers. IR Is are Internationalized Resource Identifiers, a generalisation of Uniform Resource Identifiers (URIs). These are unique machine-readable identifiers which include - but are not limited to - the resolvable URLs most Internet users are familiar with (See RFC 3987 [Duerst and Suignard 2005], RFC 2396 [Berners-Lee, Fielding, and Masinter 1998] and URI Planning Interest Group, W3C/IETF (2001) for the [lack of] distinction between URIs and URLs). Subjects in an RDF triple can be IRIs, strings or "blank" nodes leading to further triples, objects can be IRIs or "blank" nodes, and predicates are always IRIs. The IRIs serve as unique identifiers for nodes (resources) on the web and, preferably, as resolvable addresses on the World Wide Web (that is, valid locations on the global Internet that can be dereferenced to return structured data). 
From these basic building blocks, complex and flexible data structures can be created spanning multiple organisations and platforms, distributed across the Internet. The use of IRIs as unique identifiers that are also addressable allows for seamless linking together of disparate resources, and facilitates both extensibility and the use of shared vocabularies and ontologies. Structured vocabularies, in the Linked Data sense, are lists of concepts and terms each with their own IRIs and often with links describing how they relate to one another in a hierarchy or to related terms in other vocabularies. They are typically expressed using the RDF applications RDFS (RDF Schema; see Brickley and Guha 2014) for simple term lists and Simple Knowledge Organization System (SKOS; see Miles and Bechhofer 2009) for structured thesauri. Linked Data ontologies expressed using the Web Ontology Language (OWL; see Motik et al. 2012) define properties and relations that can exist between nodes, as well as the types of nodes such relations can have as subject and object (their "domain" and "range") and what such relations might entail, semantically, if anything. Vocabularies and ontologies allow different users within a domain or field to describe their data using consistent terminologies and structures. This use for IRIs also decouples attributes from identifiers, in the sense that instead of recording for example a string value, you instead reference an identifier which in turn may have multiple string labels associated with it in different languages, and possibly other attributes such as links to related (e.g., broader, narrower) terms. It also allows your data to be enriched and augmented by others simply by linking it with their own data. The rewards of applying a Linked Data model to data publication are interoperability, interconnectedness, and reusability. The paradigm facilitates citation, source verification, accessibility and reuse. Linked Data connects records within and across data sets, providing machine-readable context in a way that would otherwise be lacking, which in turn can allow unexpected connections to appear or be inferred indirectly between records. Using shared ontologies and resolvable identifiers from vocabularies and structured thesauri increase interoperability with other data sets by ensuring that records are described in a consistent way using machine-readable terms that are language-independent and common across data sets within the same field of knowledge. And links to the sources which corroborate the assertions in a record, or on which the record itself is derived provide a degree of verifiability and increase user trust.

The "open" part of LOD refers to the use of open licenses that permit the data to be used and repurposed. In most cases this in practice means the use of Creative Commons (CC) licenses and the Public Domain mark for works whose copyright has expired. The most open of the $\mathrm{CC}$ licenses is the Creative Commons Zero (CC0) license which effectively permits any use and imposes no demands on the user, and the Creative Commons Attribution (CC BY) license whose only condition for use is proper citation of the copyright holder. Use of CC0 is strongly encouraged for metadata, as 
metadata is often reused and combined with other data potentially drawn from multiple contexts and sources in such a way that citation of individual rights holders would be difficult. Otherwise, the CC BY license is generally recommended, and its requirement of proper citation makes it a good fit in academic contexts. Less open variants of the CC BY license exist with combinations of additional restrictions, such as forbidding derivative works (ND), requiring derivative works be licensed under the same terms (SA) or allowing reuse only in non-commercial contexts (NC). The latter variants are particularly problematic due to the vague definition of what counts as "commercial" which often leads to unforeseen consequences, for example precluding use in many academic and educational contexts even when allowance for such uses is intended by the rights holder; as such they are best avoided.

$\mathrm{Nb}$. that $\mathrm{CC}$ licenses do not override copyright, but work just as other licenses do, by granting permission to use material despite copyright; CC licenses simply happen to be unusually liberal in what permissions they grant. Non-CC open licenses, such as Open Data Commons licenses for databases and licenses for software (see e.g., https://choosealicense.com/) may also be applicable in other open data contexts.

FAIR principles - Findability, Accessibility, Interoperability, and Reuse for digital data - are an extension and development of the general move towards open data within academia, tangential - but beneficial - to LOD specifically. FAIR principles go beyond simply publishing data with an open license, but specifically require that it be Findable, Accessible, Interoperable, and Reusable. In practice this means taking steps to address resource discovery, long-term availability and digital preservation, structure and machine-readability, and adherence to applicable data standards, in addition to open licensing. Again, the intention is to facilitate collaboration, compatibility, and the combinations and reuse of data.

The application of LOD in general and within the heritage sector in particular embodies not just a shift in technical platforms, but more broadly a change in mindset, moving away from silos of incompatible data, meaningless distinctions between otherwise homogeneous data sets based solely on the institution or collection they happen to belong to, and towards a collaborative, distributed, standards-based, interdisciplinary machine-readable semantic web of data.

\section{Metadata aggregation}

The principles of LOD are predicated on a distributed model: a web of connected data spread across the Internet, hosted by multiple organisations, each one responsible for their own data and assertions. This web, in whole or in part, describes an open-world knowledge graph - collections of subject-predicate-object assertions describing and relating entities/resources to one another, together forming a knowledge base in the form of a network or 
graph - accessed through IRIs across multiple domains (both in the sense of subject areas and Internet domains). This web of knowledge can also be queried using federated search through SPARQL (SPARQL Protocol and RDF Query Language; see Harris and Seaborne 2013) or less commonly other query languages. It would seem on the face of it that platforms aggregating Linked Data resources - such as Europeana or SOCH (q.v.) - or those collecting large-scale Linked Data statements in a single place - such as Wikidata - ought to be antithetical to the inherently distributed nature of the Linked Data model, but this need not necessarily be the case.

For platforms such as Wikidata, it is simply a consequence of the scope of the project that it covers large numbers of triples describing metadata on topics and records more or less within the domain of the "the sum of all human knowledge" within a universal ontology. Its scope does not make it a node in web of data of any less value - on the contrary, its comprehensive nature means that in practice it functions much like a directory of identities within the world of Linked Data, providing basic metadata on vast variety of subjects, but also crucially outbound links to corresponding resources elsewhere on the web that cover more than just metadata. Thus, its broad scope and consolidated wealth of identities serve the distributed link data model.

The role of Linked Data aggregators is perhaps less obvious but no less vital, and is possibly best described as being a means rather than an end in and of itself. Both federation and aggregation attempt to solve the problem of being able to search across data sets through a single interface and receive homogenous results. While a federated search across a number of institutions/data sets is the platonic ideal of a distributed Linked Data infrastructure, there are at present practical challenges that make aggregation preferable in a number of use cases. At the performance level, federated queries face the challenge of returning results in a timely fashion, as anyone who has used an older inter-library search based on the federated Z39.50 protocol can attest. This problem is exacerbated as more endpoints are added to the network. By collecting metadata from multiple systems into a single database or index, an aggregator can provide a much more performant search across the same data sets, with the trade-off that some of the records may have become stale depending on how often the aggregator's cache is updated from the source.

Aggregation forces the source data to be mapped from its internal representation to a common data model using shared vocabularies. This occurs either at the source as the data is exposed to the aggregator for harvesting, or on the aggregator side as a part of the aggregation process. (In some cases, such as with Europeana, mappings occur on both sides of the harvesting process: first to an intermediate format, and then to a target format.) The mapping of disparate data sets from different systems with bespoke data models to a shared data model greatly simplifies crosssearch. While such mappings are also possible with a federated search 
strategy, in that case it is the individual endpoints with each respective system that are responsible for responding to queries, and thus the mapping may not be of the source data to a shared data model, but rather of the query itself to a form which better fits the capabilities of the source system to respond to it.

An aggregator also partially addresses the question of resource discovery, a problem which has yet to be satisfactorily solved for Linked Data. If you want to run a federated query, how do you find out which data sets might have data pertinent to your interests? With aggregation, this issue is delegated to the aggregator: you can search all (but also, only) the data sets harvested by the aggregator. Some progress has been made in this field, beginning with RSS feeds to provide notification of new/updated/deleted records, and more recently with Linked Data Notifications (LDN) which allow a sender to push structured RDF messages to receiving systems as changes occur.

However, the strongest argument for aggregation of Linked Data within the heritage sector is often a pragmatic one. Cultural heritage institutions (CHIs) are almost invariably short on resources, and have to accomplish a lot with a little. Publishing and maintaining a Linked Data platform is rarely part of a CHI's core mission, and frequently ranks low on its list of priorities; not because it's not considered important (although sometimes it is), but because there are so many other things that are more important and only a limited amount of money and staff to do them. Even when LOD is recognised as a priority, it can be difficult and costly to recruit or train staff with the necessary technical expertise. Aggregation thus offers a solution in such scenarios, by offloading responsibility for the LOD platform to another party. Making your data available in a way that can be harvested by an aggregator presents a lower threshold than operating your own Linked Data platform, both in terms of cost and required technical skill. Joining an aggregator shared by other similar CHIs also offers benefits in terms of networking and collaboration around shared problems and requirements.

It is to be hoped that this is not a permanent state of affairs, and that the need for aggregation of Linked Data will diminish over time, as the problems of federated query performance, resource discovery, and technical accessibility are resolved to the point that a truly large-scale federated Linked Data infrastructure no longer has any need for aggregation.

\section{Case study: SOCH}

SOCH (K-samsök in Swedish) <http://kulturarvsdata.se/> is a national aggregator for cultural heritage data in Sweden. The platform is operated by the Swedish National Heritage Board on behalf of participating and contributing data partner institutions from within the Swedish cultural heritage sector. SOCH harvests metadata records from these museums, 
archives and historic environment registers, and publishes them as LOD. The records are mapped to a common RDF-based data model, indexed, and made queryable via a web application programming interface (API). Data from $\mathrm{SOCH}$ is then in turn aggregated from the national to the European level via Europeana. Participation in and contribution of data to SOCH is free of charge and entirely voluntary on the part of the CHIs, and almost all national and county-level museums are data-providing partners. The fact that separate domain-based aggregators to Europeana exist for archives and libraries, and a historical focus on archaeological museum collections explains why museums are better-represented among SOCH partners and content than other CHIs.

At the time of writing, $\mathrm{SOCH}$ harvests and indexes data from almost 80 partner institutions from within the Swedish cultural heritage sector, who provide around 9.2 million records from 176 different data sets - in some cases discrete collections, in others multiple collections managed by a single information system. SOCH includes records covering both tangible and intangible heritage, including protected sites and monuments, historic buildings, artefacts and small finds, photographs and drawings, sound and video recordings, documents and literature, as well as historic personages, events, and more.

SOCH harvests and indexes only the metadata for records already published elsewhere; the metadata always includes a link back to the source. In cases where a record describes a digital resource such as an image, only the metadata is harvested, not the resource itself. However, the metadata contains a link to such resources where they are published, so that they can be accessed. As an aggregator, $\mathrm{SOCH}$ packages and republishes metadata from multiple sources in a more convenient - structured and searchable format, but it not itself a source for that metadata; as such, it's important that it always includes a link back to the source (and to any associated media) at the relevant $\mathrm{CHI}$.

SOCH itself offers a technical interface for machine agents via its API and the record metadata from dereferencing SOCH IRIs. A humanfriendly web interface is provided in the form of Kringla http://www. kringla.nu/ which allows users to search, browse, and view all records indexed in $\mathrm{SOCH}$, including associated media (images, audio, video), links between records, (e.g., from a photograph to the monument, artefact or person it depicts or the reverse - from a monument to all the photos that depict it), and links to the records' source. However, the combination of structured open data using a shared data model, semantic linking between records using RDF, and an API, is intended to facilitate interoperability with other platforms and to encourage use and re-use of cultural heritage data by third parties - via apps, websites, and other media, alone or in combination with other open data sets, and in ways we cannot anticipate. See Riksantikvarieämbetet (2021c) for a non-exhaustive list of applications using the SOCH data and API, including mobile apps for finding 
nearby ancient monuments, digital museum exhibitions, apps for digital storytelling in relation to cultural heritage, academic research platforms, Twitter bots, and more.

\section{Background and development}

Development on SOCH began in 2008 building on prior work to link together records from the Swedish National Heritage Board's national monuments register (FMIS) and the digital catalogue of the Swedish History Museum. The platform was developed as part of a government mandate at the Swedish National Heritage Board, and was launched in 2010. SOCH continued to receive earmarked funding from the Swedish Ministry of Culture until 2017, from which point it was financed as part of the National Heritage Board's annual budget. SOCH's launch broadly coincided with the launch of the European cultural heritage aggregator Europeana, and $\mathrm{SOCH}$ was among the first national aggregators to provide data downstream to Europeana as the national aggregator for Sweden, a role it still fulfils today.

All metadata records in $\mathrm{SOCH}$ are openly licensed as $\mathrm{CC} 0$, and for records that describe a digital resource the metadata must also include a rights statement for the resource itself; SOCH advocates for open CC licenses and public domain statements, but copyrighted works with all rights reserved are also permitted.

\section{Prerequisites and requirements}

Both CC0 licensing of the metadata and the provision of machine-readable rights statements per SOCH's rights model (Riksantikvarieämbetet 2021a) are prerequisites for partners to provide metadata to $\mathrm{SOCH}$. Other requirements that must be met by providing data partners are that records are already published online (so that SOCH can link back to the record source), and that the data is reliably maintained by an institution (so that there is a point of contact, and data management is not tied to a single individual).

In addition to this there are technical requirements necessary to enable harvesting and indexing of the data to $\mathrm{SOCH}$. SOCH harvests metadata as eXtensible Markup Language (XML; see Bray et al. 2006) using the Open Archives Initiative Protocol for Metadata Harvesting standard (OAI-PMH; see Lagoze et al. 2015), and metadata must be in the form of SOCH's RDF-based data model (Riksantikvarieämbetet 2021b). This requires firstly that the provider has a server set up that can speak the OAI-PMH protocol and respond to requests; this is typically not too onerous as there are multiple open implementations of the protocol that integrate with a variety of programming languages. Secondly, it requires a mapping from the providing institution's internal data model to the SOCH RDF data model. This component is typically much more 
demanding, requiring as it does a combination of technical programming and data modelling skills, detailed domain knowledge of the data set and associated model in question, and familiarity with SOCH's data model. The importance of such a mapping should not be overlooked, however: by requiring data to be provided according to a common model and format, we not only ensure that the data is harmonised and searchable across heterogenous data sets, but also that it is decoupled from its source format. SOCH doesn't care what model or system data partners use to manage their data, and makes no demands other than those outlined above, as long as the data has been mapped to comply with the SOCH model when it is harvested. Typically, SOCH partners are mapping data from digital collections management systems which use their own bespoke internal data models.

The requirements for providing data as a partner institution in $\mathrm{SOCH}$ are intended to be few and simple to accomplish in order to make it as frictionless as possible for institutions to publish their data for harvesting. In order to ensure that the metadata is available for reuse in as broad a variety of contexts as possible, including those where it may be combined with data from other sources or in other contexts where proper citation required by more restrictive open licenses such as CC BY may not be practical, requiring $\mathrm{CC} 0$ for metadata is more or less unavoidable. Requiring rights statements for media resources is likewise vital if users are to know if and under what conditions they may use the media. Requiring a link to the source, and a point of institutional contact are important for an aggregator republishing metadata for which it is not itself responsible, as well as for sustainability. Mapping to a common data model and the harvesting protocol used are perhaps less vital, but are nonetheless pragmatic technical necessities for homogeneity and realistic implementation within the heritage sector.

\section{Metadata harvesting}

SOCH data sets are harvested via the OAI-PMH protocol. Metadata harvests are scheduled and carried out automatically by the system, the frequency of harvesting varying according to the preferences of the providing data partner and the frequency with which the source data sets are updated; weekly harvests are typical, but some data sets are updated less often. Normally only changes since the last harvest - deletions, insertions, and updates - are requested, but in cases where the providing system does not support this facet of the protocol, the full data set is harvested every time. Harvested data is first saved to disk as RDF/XML, and then inserted into the $\mathrm{SOCH}$ database which represents records as discrete RDF/XML documents. Finally, the SOCH index is updated with the records' content. It is the SOCH index which drives the majority of the platform's functionality. The index not only enables searching of metadata in SOCH - from simple 
text searches to complex queries - but also maintains reciprocal semantic links between records.

SOCH records are published with permanent IRIs under the kulturarvsdata.se domain (kulturarvsdata is Swedish for "cultural heritage data"). These IRIs function both as record identifiers and as resolvable addresses. Depending on the format requested, dereferencing a record's kulturarvsdata.se IRI returns that record's RDF from the database as either RDF/ XML or JSON-LD (Kellogg et.al. 2020) for machine agents, or redirects to the source for that record for human-operated user-agents such as web browsers.

As part of the harvesting process, $\mathrm{SOCH}$ keeps track of records which have previously been harvested but have subsequently been removed at the source; the permanent IRIs for such records are still valid as identifiers, but return an HTTP "410 Gone" code if dereferenced (indicating that the IRI is correct but the resource has been removed; cf. "404 Not Found" where the IRI is unknown to the system).

The choice of the kulturarvsdata.se domain for SOCH IRIs rather than, for example, a subdomain of the Swedish National Heritage Board's raa. se was deliberate. Firstly, although the platform is operated by the Swedish National Heritage Board, the Board is only one of almost 80 partners represented in $\mathrm{SOCH}$, and using the raa.se domain would thus not accurately reflect the content of the platform. Secondly, decoupling the domain from the Heritage Board contributes to the longevity of SOCH records' permanent IRIs - should Swedish government agencies be restructured at some future date, for example, and the Heritage Board be dissolved or its responsibility for SOCH reassigned, ownership of the kulutrarvsdata.se domain can easily be transferred and records can keep their existing IRIs.

\section{The SOCH data model}

Within the framework of RDF, SOCH uses its own bespoke data model of defined elements, attributes/properties, and types. The model is defined as OWL under the namespace <http://www.kulturarvsdata.se/ksamsok\#> and documented on the SOCH homepage (Riksantikvarieämbetet 2021b, idem. 2020a). A number of the attributes correspond exactly to, and in some cases are interchangeable with, well-known attributes from other ontologies - most notably OWL for e.g., owl:sameAs identities, Dublin Core terms and elements for fundamental metadata, Friend-of-a-Friend (FOAF), attributes for describing people and organisations, and even some properties from the CIDOC-CRM ([ICOM] International Committee for Documentation Conceptual Reference Model). In general, however, the SOCH data model is bespoke.

Any mapping between data models inevitably leads to information loss, and this is particularly acute in the case of aggregators, where detail from the bespoke data models of the source may become blurred when the same 
data is viewed through the lens of an aggregator. Aggregation services walk a tightrope: one the one hand they must maintain a data model abstract enough that it allows data from multiple sources - sometimes across multiple disciplines - to be combined. On the other, the model must be detailed enough that it remains useful to users.

At its core the SOCH data model consists of "items" or "entities" - the records - which are described with the help of various metadata attributes. Attributes include descriptors such as itemLabel, provenance information such as parish, chronological information such as fromTime and toTime, links to related objects such as isVisualizedBy and more. Most attributes take strings or IRIs as values, conforming to the RDF subject-predicateobject model. Defined item types and their umbrella super-type for records are referred to by their IRIs, defined by SOCH (Riksantikvarieämbetet 2018a).

In cases where attributes take IRIs as values, it may be to refer to an authority record, for example when giving a thesaurus term, or to describe a semantic relation to another record. Such attributes are described as "relations", and are an important part of not only the SOCH data model but its implementation of the philosophy of Linked Data, placing records within a wider semantic context by describing their relationships to one another. Some attributes take further qualifying attributes themselves before arriving at a value, represented as blank nodes in RDF. For example, a record might have an itemName consisting of a type, e.g., "Keyword", and the name itself e.g.,"Axe".

A particular case of such multifaceted attributes and values tied to SOCH records is that of "contexts". Contexts provide a temporal and spatial dimension to the objects they describe by providing information about instances in the objects' history - although not to the same extent as a fully event-based model such as CIDOC-CRM. An object's record may have multiple contexts, each of which describes a different aspect or event in the life of the object. Like items themselves, contexts have defined types and supertypes (Riksantikvarieämbetet 2020b). For example, an artefact might have a context for its creation, containing attributes for date, place, and perhaps maker if known; it may have other contexts detailing when it changed ownership (and to whom), when/where it was used, when/where it was found and by whom, and even in some cases the occasion of its destruction.

Only a few of the basic attributes are required for a record to be valid under the SOCH data model, keeping the threshold for data provision low. At the same time, the model provides for much richer and more nuanced records where such data exists through the use of contexts, multiple attributes, reference to common authorities, and links to other records. Mandatory properties for a record are limited to protocol version, service name and organisation, source URL, item type, item name and label, lastchanged date, and license ( $\mathrm{CC} 0)$. 


\section{Authorities}

Within the realm of Linked Data interoperability, it is very often preferable to have attribute values (the "objects" of an RDF triple) that take IRIs rather than text strings, not only when referring to other records but in particular when referring to authorities for well-known things, concepts, features, categories, etc. Data partners in $\mathrm{SOCH}$ are encouraged to do this in the general case, for well-known resources. Common useful authorities within the heritage sector include inter alia the Getty Arts and Architecture Thesaurus (AAT), PeriodO for spatio-temporal chronologies, sites and monuments types thesauri such as those at Heritage Data, Virtual International Authority File (VIAF) for authors, and GeoNames and relevant national authorities for places. Linking to generic and well-known meta-authorities such as Wikidata is also useful from a general interoperability perspective. However, $\mathrm{SOCH}$ specifically provides a number of authority IRIs under the prefix <http://kulturarvsdata.se/resurser/aukt/*> which are of particular use for Swedish heritage data (Riksantikvarieämbetet 2020b). These consist of concepts integral to the SOCH data model (object types, context types, broad topic keywords, and licenses for media), geographical divisions for Sweden (parishes, municipalities, counties, provinces, and countries and continents linked against GeoNames), as well as concepts specific to the study of runes and runic inscriptions.

\section{The SOCH API}

Metadata records in $\mathrm{SOCH}$ can be accessed by dereferencing the resource's IRI, but for most practical applications, the main point of access to the content in SOCH is its web API (Riksantikvarieämbetet 2019), the technical interface by which programs can query the index and receive structured data in response. The API provides a number of methods for searching and retrieving records, as well as for finding and following links between records, and options for formatting the metadata output. There are also helper methods for aggregate searches (e.g., statistics on records matching search criteria or listing the frequency of certain property values in the index), auto-complete and stemming for search terms, and for other information about the contents of the index. Search indices are delineated by attribute according to the SOCH data model (Riksantikvarieämbetet 2018b). The search methods use the Contextual Query Language (CQL) standard for the specification of queries and indexes. This allows for faceted search across multiple indices, as well as for complex searches using Boolean AND/OR/ NOT operators.

The default output of the search API is an XML result set encapsulating the full RDF/XML documents of matching records. However, the API allows clients to specify other preferred output formats; JSON may be substituted for XML, and JSON-LD for RDF/XML as when dereferencing records' IRIs. 


\section{User-generated content}

Parallel to - but separate from - SOCH is a second system that allows third parties to contribute semantic links of their own between $\mathrm{SOCH}$ records or between $\mathrm{SOCH}$ records and external resources on the semantic web such as identities maintained by other organisations, vocabulary terms or related records elsewhere on the web. This system is called the UGC Hub ("UGC" for "User-Generated Content") and it has its own index and API. Usergenerated links may be queried or added via the API or via the Kringla web interface http://www.kringla.nu/. The UGC Hub allows records in SOCH to be enriched and augmented with additional links not provided by SOCH data partners, while keeping such third-party links separate and distinct from the metadata curated by those institutions. User-contributed links typically follow the property and relation types of the SOCH data model, relating records to one another or to external identities and vocabularies. However, as the links should take the form of fully qualified IRIs, they may in theory include any valid RDF property type (although clients, such as Kringla, may not understand all such properties). At the time of writing the UGC Hub boasts an index of 2.7 million links. Among other benefits, the UGC Hub has allowed for much tighter integration between $\mathrm{SOCH}$ and other sites, thanks to automated agents maintaining links between SOCH records and their corresponding IRIs at Europeana, Wikidata, and Wikipedia, as well as associated media on Wikimedia Commons. These added links provide identity relations with records describing the same thing on other platforms, contextual relations, additional visual media, and starting points for further reading or research.

\section{Applications}

The open nature of both the content of the metadata in SOCH and its API was from the very start intended to foster and encourage the creation of third-party applications to use the data and combine it with data from other sources. It was and is felt that the Swedish National Heritage Board's role is to ensure that the heritage data is made available in a way that ensures that it is accessible, reusable, and flexible, and to maintain the SOCH platform and its technical interface - not to create multitudes of websites and apps for various platforms to satisfy every possible use-case and niche interest. Indeed, the de-facto default web interface to $\mathrm{SOCH}$, Kringla, was originally intended simply to be a proof-of-concept showcase for the data, presenting it in a more accessible manner than the RDF/XML-based SOCH interface.

Aside from the Kringla website and a now-defunct proof-of-concept Kringla app for Android phones, the National Heritage Board has also developed prototype applications to examine alternative non-search-based methods of exploring the records, collections and objects published through $\mathrm{SOCH}$ using "generous interfaces" (Generous Interface Fashion; Kringla 
Visualized). Furthermore, a new research platform for runes and runic inscriptions has been developed using $\mathrm{SOCH}$ in collaboration with Uppsala University (Runor). However, the overwhelming majority of applications using and building upon the open data in SOCH and its API have been created by third parties, from mobiles apps to mashups with other data sets, and even programming frameworks designed to make it easier for other developers to use SOCH from their programming language of choice (see Riksantikvarieämbetet 2021c for a list of examples). It is interesting to note that in some cases, museums providing their collections data to $\mathrm{SOCH}$ have built interfaces to those collections using $\mathrm{SOCH}$, rather than the collections management software (CMS) in which their master data is managed. In some cases this is because SOCH offers a more convenient API than their in-house CMS (or because the CMS offers no API at all) but it also illustrates the tangible benefits of Linked Data through $\mathrm{SOCH}$, as objects from one collection may be enriched by links to objects from another collection or institution - for example if related objects are at different museums or one museum has photographs or other documentation depicting or describing artefacts from another. In a very real sense, publishing collections as Linked Data can enable institutions to get more out than they put in. A good example of such applications is the School Posters digital exhibition from the Museum of Gothenburg, http://www.stadsmuseetsskolplanscher.se/.

\section{Problems and challenges}

The SOCH platform in its current state is however not without its weaknesses, and it would be remiss not to address them in this case study - particularly in the interests of promoting best practice and of warning others not to repeat our mistakes.

Firstly, SOCH's technical platform itself, while sturdy and reliable, shows its age at $10+$ years, in particular in the way it handles RDF data. SOCH is not a triplestore, and doesn't treat its RDF as a graph; rather, each record is held and indexed as an RDF/XML document. This causes some limitations in how the platform can be used and what kinds of data it can store. For example, it is not possible for two institutions or data sets to provide complementary records for the same object using the same IRI - each record must have its own IRI - and it is not possible to nest record IRIs within the same document - each must be the root element of its own document. Were $\mathrm{SOCH}$ a true triplestore with an RDF graph, these limitations would not exist.

The SOCH API, too is showing its age. In the time since it was first designed, web services have coalesced around REST-based APIs (Representational State Transfer; see Fielding 2000) serving JSON as a de-facto standard interface paradigm, which SOCH does not conform to. (SOCH can serve JSON-LD, but responds with RDF/XML by default, and does not support a RESTful interface at all.) This may present an off-putting 
barrier to use for modern web developers. On the other hand, SOCH does not provide a SPARQL endpoint interface of the kind expected by Linked Data users either (partly as a direct consequence of the platform architecture mentioned above), thus failing to satisfy two of our main developer target audiences.

As mentioned above, the SOCH data model is bespoke for the platform. At the time it was developed, Linked Data had not been widely deployed in the heritage sector, and while some standards did exist for linked heritage data, they were not yet in widespread use. Now, however, standards such as CIDOC-CRM and the Europeana Data Model (EDM) are well-known within the sector, and from an interoperability standpoint it would make more sense to use them - or a compatible application of them - rather than using custom types and attributes that are unique to the platform. The data model also makes poor and sometimes curious use of the Linked Data capabilities of RDF. A number of attributes take IRIs as values but type them as strings; in other cases, values which are IRIs are split into prefix and "slug" (suffix) halves which are assigned to separate attributes, defeating the point of having the link in the first place. Perhaps most seriously, the data model makes no attempt to address the "httpRange-14" issue of Linked Data (W3C 2002), that of distinguishing between the IRI of a physical object and the digital record that describes it; the SOCH data model merges the two under a single IRI, and resolves the fact that the object and the record may have separate and distinct values for attributes such as "date created" by having separate attributes rather than separate entities.

As described earlier, records in SOCH are assigned IRIs by their providing institutions as part of the mapping and harvesting process, often based on the internal IDs used by the providers' collections management system. Having IRIs tied to both institutions and their internal systems poses challenges in terms of long-term sustainability as both are potentially subject to change. This has been addressed recently with support for relations describing changed IRIs (dcterms:replaces), allowing data providers to change their IRIs if necessary, providing a new identity relation between the old and the new IRIs. SOCH will redirect requests for the old IRI to the new one that replaces it, and transitively treats relations that apply to one as if they also applied to the other.

Finally, the quality of SOCH as a platform is in large part dependent on the quality of the metadata it aggregates, and the quality of this metadata is highly variable between heritage institutions. Despite being a platform predicated on Linked Data, links between records are less common than we would like, and links between data sets and providers even less common. Attribute values are almost always provided as strings rather than as IRIs linking to terms from a shared vocabulary. The potential offered by Linked Data is, in other words, not always exploited to its fullest. Data provided by SOCH partners usually comes from collection management systems originally intended for internal (rather than public) use, and the data itself may 
have been digitised from older analogue records that were taken with a different purpose in mind; this also contributes to the variability in metadata quality. In most cases it is not possible for SOCH to check whether incoming data is correct; however, in some cases, such as copyright status, an independent verification can be made: data partners can check to see if their provided data sets contain any obviously incorrectly licensed records using an online tool at https://riksantikvarieambetet.github.io/ksamsok-dataportal/. Typical errors include claiming copyright over media that has fallen to the public domain, media licensed with a CC BY license but with no data on to whom attribution should be given, and photographs dated both to the future and to before the invention of photography. Aside from these cases, however, SOCH is dependent on data partners to ensure data quality.

When SOCH was first developed, the threshold for data provision was deliberately kept as low as possible in order to make it easy for CHIs to be able to provide data from their collections. Aside from licensing and a link to the published record, no conditions were set as to data quality. It was deemed more important to enable institutions to publish their records through the platform regardless of the metadata quality, on the understanding that metadata quality could always be improved upon at a later date, and that no data set will ever be "perfect". This made uptake easier, but has meant that the quality of metadata in the SOCH index can vary wildly, from rich records with detailed metadata and semantic links to records which are barely more than empty placeholders for the artefacts or resources they represent. The threshold for data provision is still too high for some smaller resource-starved heritage institutions, and efforts are now being made to explore alternative methods of data harvesting that may enable them to become partners in SOCH using their existing technical infrastructures, for example by harvesting from the APIs of CHI's content management systems and mapping that to the $\mathrm{SOCH}$ data model, harvesting unstructured or semi-structured data from CHI's web pages or by harvesting a simplified record description based on standard generic Linked Data attributes such as Dublin Core, schema.org, and FOAF.

\section{Reflections}

The application of the LOD paradigm in SOCH has benefited both the heritage sector and the general public by making search possible across multiple institutions, collections and data sets, by making heritage data available to freely reuse and develop in ways that were not possible before, by integrating and linking together records across collections, placing them in a wider context, and by offering users the opportunity to themselves engage with and contribute to the Linked Data platform via the UGC Hub.

Over its, at the time of writing, ten-year lifespan, SOCH has had significant success in opening up Swedish heritage data from institutional silos and linking it together, especially for institutions which otherwise lack the 
resources to publish their data in a machine-readable and searchable format. All metadata records harvested to $\mathrm{SOCH}$ are licensed as $\mathrm{CC} 0$, and all media resources must be marked up with rights statements, making terms of reuse clear. Semantic links between records are strongly encouraged, as are links out to external resources, vocabularies and authority files. Links to Wikipedia, Wikimedia Commons, Wikidata and bibliographic records at the Swedish National Library are particularly useful for connecting the aggregated records with the wider semantic web, providing a wider context, references and a jumping-off point for further research through onward links. SOCH data and its open API are used by numerous third-party applications to present, explore and reuse Swedish cultural heritage data in a variety of ways, both on its own and in combination with other open data resources; from serious academic research platforms to smartphone apps for casual use, to more trivial uses in games and Twitter bots. Despite their different uses, contexts, and target audiences, all these applications are built on the same linked open heritage data and API. Material in SOCH is seen and used more broadly and by more people than it otherwise would be.

The SOCH platform has served Swedish heritage well since its inception in 2008, and there are now plans to develop it further in order to better serve the needs of modern users and developers, as well as to better align with modern heritage data standards and address some of the challenges described above. It is hoped that the redeveloped SOCH will not be the only platform to learn from the experience of the past 13 years, and that it may serve as inspiration for other LOD heritage platforms in future.

Ultimately, in the longer term, it is to be hoped that the need for aggregators such as SOCH and Europeana will become unnecessary as CHIs find themselves more able to publish and reliably maintain their own standards-based LOD platforms, and the problems of resource discovery (e.g., widespread adoption of LDN) and responsive federated search are mitigated. Indeed, some institutions, such as the Swedish National Library, have already gone this route, publishing via a robust Linked Data platform and eschewing aggregation. Until this approach becomes more widespread, however, aggregators fill a valuable role, centralising search via a single API endpoint and providing timely responses, enforcing adherence to a homogenous data model, and relieving CHI of some of the economic and technical burdens concomitant with maintaining such a platform. The challenges facing linked open cultural heritage data in the coming years are ones of data quality, skills and training among heritage professionals, funding, and adoption. Of these, data quality is in some ways both the most pressing and the easiest to make swift progress in: despite best efforts, linked open heritage data is still not especially linked, with string values carried over from other systems still being published instead of proper links to other records or to vocabularies/authorities. Updating such references is in itself not difficult but would at a stroke vastly improve the usefulness and value of the linked heritage data web by enriching and contextualising the data. 


\section{Conclusions}

This chapter has given an introduction and overview of elementary LOD and metadata aggregation on both a philosophical and a technical level, introducing key concepts and standards, as well as giving a practical example in the form of the SOCH Linked Data aggregator platform. We have shown that the LOD model solves the problems of closed data platforms within the heritage sector - both in terms of licensing and reuse, and interoperability and accessibility - posed by the widespread data silo model, by leveraging the possibilities of connecting data sets together over the web.

Covering the background of LOD and metadata aggregation, we have seen how data can be more richly and flexibly described in a machinereadable manner by using semantically meaningful links to describe properties and relations according to a graph-based model. We have also seen how this can increase interoperability across data sets, especially with the use of shared structured vocabularies for terms and ontologies for structure and meaning. By publishing data in this way, assertions from different data sets describing the same or related subjects can complement one another, and can be expanded upon by others publishing their own Linked Data.

The SOCH Linked Data platform and aggregator for $\mathrm{SOCH}$ was presented as a concrete example of a large and successful platform showing how some of these principles can function in practice. However, while advanced for its time in 2008, SOCH has failed to keep up with developing standards within LOD. Its use of a bespoke data model and web API now serve as a hindrance to greater interoperability and integration with the wider semantic web. We also saw how metadata aggregation can work to in tandem with the goals of Linked Data: despite the fact that the distributed model of Linked Data might seem counter to aggregation's essential centralisation, in fact aggregated Linked Data works well when circumstances otherwise preclude the fully distributed semantic web data utopia that LOD aims to create.

Reflecting on the glimpse into the state of linked open cultural heritage data presented here, we have seen that the open, connected publication model that LOD advocates is beneficial for the sector and users. Openly licensed data is able to reach more people simply by dint of ease of publication and proliferation via copying on the web, and is not just accessible by (re)usable. Linked Data is more useful, both to researchers and casual users, providing as it does the potential for citable sources, context, languageindependent shared terminologies and greater interoperability beyond a single data set or source, within a distributed hypermedia platform.

The future is not without challenges however. Linked Data benefits greatly from wide adoption - the more data sets that are published as LOD, the more links there are between data sets (and data using the same vocabularies and ontologies), enriching other data sets indirectly. However, there are barriers to wider adoption within heritage, particularly a lack of 


\section{Marcus Smith}

domain knowledge and, crucially, resources, in a sector that is often already stretched thin and has to prioritise.

It is nonetheless to be hoped, however, that the LOD paradigm will continue to flourish within the cultural heritage sector - perhaps connecting via SOCH or Europeana, or inspired by their example - and that more institutions will expose their data using RDF and applicable shared Linked Data standards.

\section{Glossary}

AAT: Getty Arts and Architecture Thesaurus; a structured Linked Data vocabulary for artistic terms, styles, periods, object types etc. http:// vocab.getty.edu/

CHI: Cultural Heritage Institution

CIDOC-CRM: The CIDOC Conceptual Reference Model, an eventoriented ontology for cultural heritage with a focus on museum collections. http://cidoc-crm.org/cidoc-crm/

CQL: Contextual Query Language, a simple index-based query language with Boolean logic originally intended for use in library contexts but found in many search applications; maintained by the Library of Congress. https://www.loc.gov/standards/sru/cql/spec.html

Creative Commons: A non-profit organisation promoting free culture and set of widely-used open attribution-based licenses for creative and cultural works. https://creativecommons.org/

CRMarchaeo: An extension to the CIDOC-CRM adding properties for archaeological methods and concepts. http://www.cidoc-crm.org/ crmarchaeo/

Dublin Core: A set of 15 fundamental metadata properties (later expanded) widely used and accepted as a baseline for metadata. http://purl.org/dc/ elements/1.1/; http://purl.org/dc/terms/

EDM: The Europeana Data Model, a data model for cultural heritage data used by the Europeana aggregator Europeana. http://www.europeana. eu/schemas/edm/

Europeana: A European digital cultural heritage aggregator operational since 2008 and with collections data from more than 3,000 CHIs, operated under the auspices of the European Commission. https://www. europeana.eu/

FAIR: A set of principles for Findable, Accessible, Interoperable and Reusable digital data, complementary to Linked Open Data and movements such as Free Software, but with a focus on academia and scientific data. https://www.go-fair.org/fair-principles/

FOAF: Friend-Of-A-Friend; a Linked Data schema for describing people, organisations and social networks. One of the earliest Linked Data ontologies, it is in widespread use. http://xmlns.com/foaf/0.1/ 
Generous Interface Fashion: An experimental application built on the SOCH API and data as a prototype "generous interface", allowing users to explore a digital collection rather than having to search. https:// riksantikvarieambetet.github.io/Generous-Interface-Fashion/

GeoNames: An authority for geolocated place names and geographical divisions (countries, provinces, cities etc). http://www.geonames.org/

Heritage Data: A set of Linked Data SKOS vocabularies and thesauri from the UK heritage bodies, including monuments types thesauri, https://heritagedata.org/live/schemes

JSON-LD: A variant of the JavaScript Object Notation data format with support for Linked Data; a serialisation format of RDF; see Kellogg et al. 2020.

Kringla: A web application built on SOCH allowing users to search, browse and view records in $\mathrm{SOCH}$, including associated media, links between records and out to the broader web etc.; https://www.kringla. nu/

Kringla Visualized: A proof-of-concept application for visualising statistics and trends in the properties of records in SOCH; https:// riksantikvarieambetet.github.io/Kringla-Visualized/index_en.html

K-samsök: See SOCH

LDN: Linked Data Notifications, a protocol for pushing RDF-based messages from a sender to a receiver, for example as a notification of changes to a data set; https://www.w3.org/TR/ldn/

OAI-PMH: Open Archive Initiative Protocol for Metadata Harvesting; see Lagoze et al. 2015.

Open Data Commons: https://opendatacommons.org/licenses/odbl/1-0/

OWL: Web Ontology Language; an RDF ontology for describing RDF ontologies; see Motik et al. 2012

PeriodO: A linked open data gazetteer for historical and archaeological chronological periods, bounded both temporally and geographically. https://perio.do/

RDF: Resource Description Framework, the meta-model for linked open data and the semantic web, comprised of three-part statements called triples; see Schreiber and Raimond 2014

RDFS: RDF Schema, a set of RDF types and predicates for describing data schemas and vocabularies; see Brickley and Guha 2014

REST: Representational state transfer, a method of designing web APIs using hypertext transfer protocol semantics; see Fielding 2000.

RSS: RDF Site Summary, or alternatively Really Simple Syndication, a method of providing a data feed of new or changed records (or blogposts, or podcasts...) over the web; https:/www.rssboard.org/rss-specification

Runor: A digital research platform for Nordic runic inscriptions built using SOCH, in collaboration with Uppsala University; https://app.raa. se/open/runor/ 
schema.org: A widely adopted standard for embedding generic structured semantic metadata in web pages. https://schema.org/

SKOS: Simple Knowledge Organization System, a set of RDF types and predicates for describing multihierarchical structured vocabularies as Linked Data; see Miles and Bechhofer 2009

SOCH: Swedish Open Cultural Heritage (in Swedish K-samsök) the Swedish national cultural heritage aggregator and linked open data platform, operated by the Swedish National Heritage Board. http:// kulturarvsdata.se/

VIAF: Virtual International Authority File. a set of aggregated authorities for bibliographic entities such as authors, works etc. collected from national libraries across the world; http://viaf.org/

Wikidata: Crowd-sourced structured linked open data from Wikimedia; http://www.wikidata.org/

Wikimedia Commons: Crowd-sourced open media from Wikimedia; https:/commons.wikimedia.org/

Wikipedia: Crowd-sourced open knowledge from Wikimedia; https:// www.wikipedia.org/

Z39.50: A federated search protocol that used to be popular for interlibrary resource discovery; standard maintained by the Library of Congress; https://www.loc.gov/z3950/agency/

\section{Bibliography}

Berners-Lee, Tim, Roy Fielding, and L. Masinter 1998. RFC 2396: Uniform Resource Identifiers (URI): Generic Syntax. Internet Engineering Taskforce. http://tools. ietf.org/html/rfc2396

Berners-Lee, Tim 2006. Linked Data. Cambridge, MA, World Wide Web Consortium (W3C). http://www.w3.org/DesignIssues/LinkedData.html

Bray, Tim; Jean Paoli, C. M. Sperberg-McQueen, Eve Maler, François Yergeau, and John Cowan (eds.) 2006. XML 1.1, 2nd edition. Cambridge, MA, W3C Recommendation. https://www.w3.org/TR/2008/REC-xml-20081126/

Brickley, Dan and R.V. Guha (eds.) 2014. RDF Schema 1.1. Cambridge, MA, World Wide Web Consortium (W3C). http://www.w3.org/TR/rdf-schema/

Duerst, Martin and Michel Suignard 2005. RFC 3987: Internationalized Resource Identifiers (IRIs). Internet Engineering Taskforce. http://tools.ietf.org/html/rfc3987

Fielding, Roy Thomas 2000. "Architectural Styles and the Design of Network-based Software Architectures.” PhD diss., University of California, Irvine. http://www. ics.uci.edu/ fielding/pubs/dissertation/rest_arch_style.htm

Harris, Steve and Seaborne, Andy (eds.) 2013. SPARQL 1.1 Query Language. http:// www.w3.org/TR/sparq111-query/

Kellogg, Gregg, Pierre-Antoine Champin, Dave Longley, Manu Sporny, and Markus Lanthaler. 2020. JSON-LD 1.1: A JSON-based Serialization for Linked Data. Cambridge, MA, World Wide Web Consortium (W3C). http://www.w3.org/TR/json-ld/

Lagoze, Carl, Herbert Van de Sompel, Michael Nelson, and Simeon Warner (eds.) 2015. The Open Archives Initiative Protocol for Metadata Harvesting. http://www. openarchives.org/OAI/openarchivesprotocol.html 
Miles, Alistair and Sean Bechhofer (eds.) 2009. SKOS Simple Knowledge Organization System Reference. Cambridge, MA, World Wide Web Consortium (W3C). http://www.w3.org/TR/skos-reference

Motik, Boris; Patel-Schneider, Peter F. and Bijan Parsia (eds.) 2012. OWL 2 Web Ontology Language: Structural Specification and Functional-Style Syntax. 2nd edition. Cambridge, MA, World Wide Web Consortium (W3C). http://www. w3.org/TR/owl-syntax

Riksantikvarieämbetet. 2018a. Objektstyper. https://www.raa.se/hitta-information/ k-samsok/att-anvanda-k-samsok/objektstyper/

Riksantikvarieämbetet. 2018b. Index för statistic/facet. https://www.raa.se/hittainformation/k-samsok/att-anvanda-k-samsok/index-for-statistic-facet/

Riksantikvarieämbetet. 2019. Metoder. https://www.raa.se/hitta-information/k-samsok/ att-anvanda-k-samsok/metoder/

Riksantikvarieämbetet. 2020a. Relationstyper. https://www.raa.se/hitta-information/ k-samsok/att-anvanda-k-samsok/relationstyper/

Riksantikvarieämbetet. 2020b. Auktoriteter. https://www.raa.se/hitta-information/ k-samsok/att-anvanda-k-samsok/auktoriteter/

Riksantikvarieämbetet. 2021a. Rättigheter. https://www.raa.se/hitta-information/ k-samsok/att-anvanda-k-samsok/rattigheter/

Riksantikvarieämbetet. 2021b. Protokoll och parametrar. https://www.raa.se/hittainformation/k-samsok/att-anvanda-k-samsok/protokoll-och-parametrar/

Riksantikvarieämbetet. 2021c. Exempel på användning av K-samsök. https://www. raa.se/hitta-information/k-samsok/att-anvanda-k-samsok/exempel-paanvandning-av-k-samsok/

Schreiber, Guus and Yves Raimond (eds.) 2014. RDF 1.1 Primer. Cambridge, MA, World Wide Web Consortium (W3C). http://www.w3.org/TR/rdf11-primer/

URI Planning Interest Group, W3C/IETF. 2001. URIs, URLs, and URNs: Clarifications and Recommendations 1.0. https://www.w3.org/TR/uri-clarification/

W3C. 2002. httpRange-14: What Is the Range of the HTTP Dereference Function? W3CTAG Issues List. https://www.w3.org/2001/tag/issues.html\#httpRange-14 